
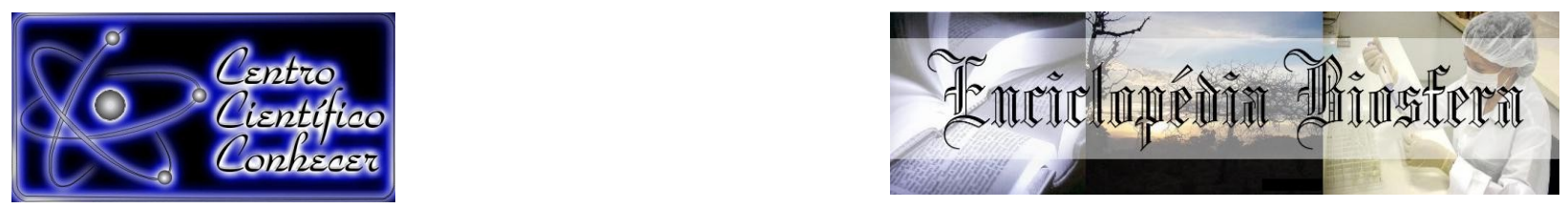

\title{
AVALIAÇÃO DO PERFIL DOS EGRESSOS DO CURSO DE BIOTECNOLOGIA DA UNIVERSIDADE FEDERAL DE GOIÁS E SUAS PERSPECTIVAS NO MERCADO DE TRABALHO
}

\footnotetext{
Elza Barbosa Marangoni ${ }^{1}$, Isabelle Guedes Porto ${ }^{1}$, Aline Menegat de Araújo ${ }^{1}$, Letícia Ferreira Campos ${ }^{1}$, Renan Xavier Ribeiro ${ }^{1}$, Thiago Lopes Rocha ${ }^{2}$.

${ }^{1}$ Graduandos do curso de Biotecnologia da Universidade Federal de Goiás (UFG), Goiânia - GO, Brasil.

${ }^{2}$ Professor Doutor do Instituto de Patologia Tropical e Saúde Pública (IPTSP) da Universidade Federal de Goiás (UFG) (thiagorochabio20@gmail.com), Goiânia GO, Brasil.
}
Recebido em: 06/04/2018 - Aprovado em: 10/06/2018 - Publicado em: 20/06/2018 DOI: 10.18677/EnciBio_2018A107

\begin{abstract}
RESUMO
O curso de Biotecnologia da Universidade Federal de Goiás (UFG) é responsável por capacitar profissionais multidisciplinares com foco na área da saúde. Embora a primeira turma de Biotecnologia da UFG tenha se formado em 2014, ainda não estão disponíveis pesquisas relacionadas à visão desses egressos e suas experiências profissionais. Nesse sentido, o objetivo deste trabalho foi avaliar o perfil dos egressos e a percepção dos mesmos em relação ao curso e ao mercado de trabalho. A pesquisa foi realizada através de um questionário online, na qual foi possível concluir que a inserção dos egressos no mercado de trabalho ainda é um desafio para o Biotecnologista, pois as empresas e instituições de pesquisa ainda não conhecem o perfil desse profissional e suas habilidades. Além disso, nota-se a importância de projetos de divulgação e extensão do curso e do profissional em Biotecnologia, para que este possa ser valorizado pela sociedade e o mercado de trabalho. Tais conclusões são úteis tanto para os estudantes atuais do curso de Biotecnologia quanto para futuros discentes. Este estudo também se faz importante para acompanhar o desenvolvimento de um novo profissional, a formação de sua trajetória no mercado de trabalho, bem como incentiva o reconhecimento da profissão.
\end{abstract}

PALAVRAS-CHAVE: Biotecnologista, egressos, ensino superior.

\section{EVALUATION PROFILE OF GRADUATES IN BIOTECHNOLOGY BY THE FEDERAL UNIVERSITY OF GOIÁS AND THEIR PERSPECTIVES IN THE JOB MARKET}

\begin{abstract}
The course of Biotechnology of the Federal University of Goiás (UFG) is responsible for capacitate multidisciplinary professionals focused in the health area. Although the first class of Biotechnology in the UFG has formed in 2014, there aren't yet available research related to the sight of these graduates and their professional experiences. In this sense, the objective of this work was to evaluate graduates profile and the ENCICLOPÉDIA BIOSFERA, Centro Científico Conhecer - Goiânia, v.15 n.27; p. 212 2018
\end{abstract}


perception of them in relation to the course itself and job Market. The research was made through an online questionnaire, in which was possible to conclude that the insertion of the graduates in job Market still a challenge for the biotechnologist, because companies and research institutes haven't yet known the professional profile of them and their abilities. Furthermore, notice the importance of disclosure projects and popularization of the course and professional in Biotechnology, in order that can receive its due recognition. These conclusions are useful for both current Biotechnology students and future students. This study is also important to accompany the development of a new professional, the formation of his trajectory in the labor market, as well as encouraging recognition of the profession.

KEYWORDS: biotechnologist, graduates, higher education.

\section{INTRODUÇÃO}

O curso de Biotecnologia da Universidade Federal de Goiás (UFG) foi criado em 2009 pelo Instituto de Patologia Tropical e Saúde Pública (IPTSP) e sua primeira turma ingressou em março de 2010. O curso possui duração de quatro anos e é responsável por formar profissionais multidisciplinares e capazes de desenvolver processos e produtos com foco na área da saúde. A matriz curricular é abrangente e envolve conhecimentos em Microbiologia, Imunologia, Parasitologia, Patologia, Bioinformática e Saúde Pública, além de contar com disciplinas optativas e de núcleo livre que permitem ao estudante direcionar o seu aprendizado para diferentes áreas da Biotecnologia, tal como saúde, industrial, ambiental ou agropecuária. São oferecidos também conhecimentos sobre Gestão e Empreendedorismo, essenciais para capacitar o profissional para atuar em pesquisa, desenvolvimento e inovação, conforme descrito no Projeto Pedagógico do curso (DE OLIVEIRA et al., 2017).

Biotecnologia é uma área multidisciplinar que utiliza organismos vivos ou parte deles, como células e moléculas para o desenvolvimento de novos produtos e resolução de problemas. A Biotecnologia tem importância em vários setores da sociedade, promovendo uma verdadeira revolução no tratamento de doenças, na reprodução de espécies vegetais e animais, na melhoria de alimentos e contribui para o desenvolvimento sustentável na recuperação e tratamento de resíduos químicos (BRUNACCI, 2005). De acordo com Ernst e Young (2014), companhias de centros de Biotecnologia nos Estados Unidos, Europa, Canadá e Austrália geraram receitas de US\$ 98,8 bilhões em 2013. Os impactos da Biotecnologia não afetam apenas a esfera econômica, mas também atingem fatores sociais, institucionais, ambientais e até políticos (OCDE, 2014).

Apesar de ter grande potencial, o Brasil ainda não mostra indicadores significativos de incorporação da Biotecnologia em produtos e processos em escala industrial (ABDI, 2009). Isso indica, dentre outros fatores, a falta de profissionais especializados. Ainda segundo ABDI (2009), um diagnóstico conduzido no âmbito do projeto Iniciativa Nacional de Inovação em Biotecnologia (INI-Biotecnologia), envolvendo participantes do governo, academia e indústria, apontou que as maiores dificuldades da cena atual com relação à dimensão "recursos humanos" na área de Biotecnologia são a capacitação, atração e fixação de talentos.

Atualmente a profissão de Biotecnologista não é regulamentada no país, sendo assim, o profissional formado em Biotecnologia não possui respaldo do conselho federal ou regional para garantir o seu devido reconhecimento pelos órgãos públicos e privados. Sem a regulamentação, fica difícil definir o perfil deste profissional, pois cada universidade acaba conduzindo a graduação de uma maneira diferente (LINABIOTEC, 2016). Em novembro de 2015 iniciou-se o processo para a 
aprovação do projeto de lei PL3747/2015, que dispõe sobre regulamentação da profissão de Biotecnologista e cria os Conselhos Federais e Regionais de Biotecnologia (BRASIL, 2015).

Nesse contexto, a percepção dos egressos mostra-se importante no acompanhamento da trajetória do profissional e deve ser vista como ferramenta de controle estratégico e operacional (ESPARTEL, 2009). Embora a primeira turma de Biotecnologia da UFG tenha se formado em 2014, ainda não estão disponíveis pesquisas relacionadas à visão dos egressos e suas experiências profissionais. Nesse sentido, o objetivo deste trabalho foi avaliar o perfil de atuação dos egressos e a percepção dos mesmos em relação ao curso e ao mercado de trabalho, expor o cenário enfrentado por estes profissionais e gerar um panorama para os atuais e futuros graduandos em Biotecnologia. Este trabalho é pioneiro na avaliação do perfil de egressos dos cursos de graduação em Biotecnologia, tornando-se um estudo relevante para melhor compreensão do processo de ensino-aprendizagem e formação do Biotecnologista no Brasil. Além disso, os dados apresentados fazem parte de um programa de avaliação continuada dos egressos em Biotecnologia, possibilitando estudos futuros sobre a temática.

\section{MATERIAL E MÉTODOS}

A coleta de dados foi realizada através do método survey, com a aplicação de um questionário online via Google Forms. O questionário foi destinado a todos os profissionais formados até 2017 (76 egressos) do curso de Biotecnologia da UFG e ficou disponível do dia 04 de junho de 2017 até 04 de agosto de 2017. Constavam neste questionário questões que tinham como objetivo caracterizar o perfil dos egressos, suas experiências, opiniões e perspectivas no mercado de trabalho.

Por meio da colaboração da coordenação do curso, foi realizado um levantamento dos egressos e em seguida feito o contato direto com os mesmos, apresentando um convite para participação na pesquisa e preenchimento do questionário enviado. Os dados coletados não expunham o participante quanto ao seu nome e nem documento pessoal ou matrícula, preservando a identidade do participante e permitindo assim uma análise crítica e imparcial.

A pesquisa do tipo survey é definida como um método de obtenção de dados ou informações sobre características, ações ou opiniões de determinado grupo de pessoas, por meio de um instrumento de pesquisa, normalmente um questionário (PINSONNEAULT; KRAEMER. 1993). Ao utilizar ferramentas virtuais pudemos aumentar o alcance de nossa pesquisa, tendo em vista que muitos egressos se encontram hoje em diversas regiões do país. A escolha dessa metodologia se deu pela praticidade e rapidez que o método proporciona.

\section{RESULTADOS E DISCUSSÃO}

O questionário online permitiu a participação de 46 egressos, os quais representam $60 \%$ do total de egressos do curso de Biotecnologia da UFG.

\section{O perfil dos egressos em Biotecnologia}

Conforme mostrado na Figura 1, nota-se um perfil de profissionais jovens, a maioria com menos de 24 anos (62,2 \%), sendo a frequência de homens (46.7 \%) e mulheres (53.3 \%) semelhante, o que mostra que as mulheres estão cada vez mais conquistando espaço na universidade e na ciência, pois, historicamente, a ciência sempre foi vista como uma atividade realizada majoritariamente por homens (LETA, 2003). 


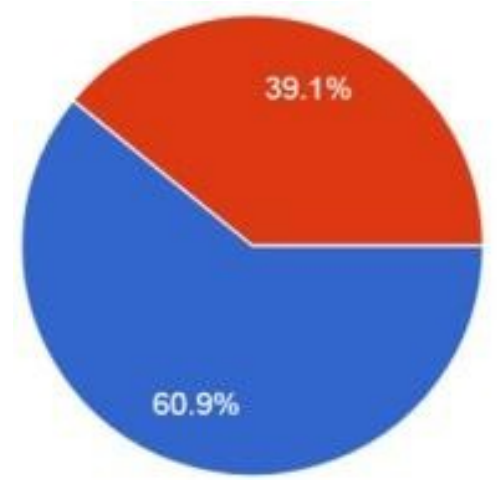

FIGURA 1: Idade dos egressos do curso de Biotecnologia da UFG.

Fonte: Elaborado pelos autores com base na aplicação dos questionários

Notou-se, também, que $47.8 \%$ dos participantes da pesquisa concluíram a graduação em quatro anos, enquanto $43,5 \%$ completaram a graduação em cinco anos e apenas $8,7 \%$ concluíram em seis anos. Não houveram participantes que alegaram ter concluído a graduação em sete anos, como mostra a Figura 2.
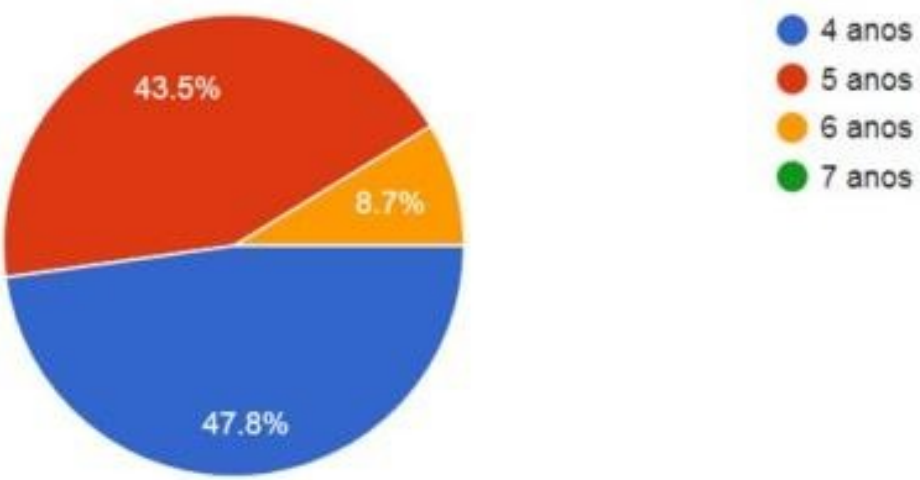

FIGURA 2: Tempo de conclusão de curso dos egressos em Biotecnologia da UFG.

Fonte: Elaborado pelos autores com base na aplicação dos questionários

O curso tem uma duração mínima de quatro anos, e máxima de seis. Nota-se que, mesmo com a grade curricular extensa (carga horária total 3.652 horas) (DE OLIVEIRA et al., 2017), a maioria dos graduados conseguiu fazer o curso em quatro ou cinco anos. Aqueles que completaram o curso em mais tempo justificam isso devido a realização do intercâmbio pelo programa ciências sem fronteiras durante a graduação.

\section{Os egressos e o mercado de trabalho}

Quando questionados quanto a receptividade do mercado de trabalho, baseado em suas experiências profissionais, a grande maioria dos entrevistados (91.3 \%) considera que o mercado de trabalho ainda não é receptivo aos Biotecnologistas, conforme se observa na Figura 3. 


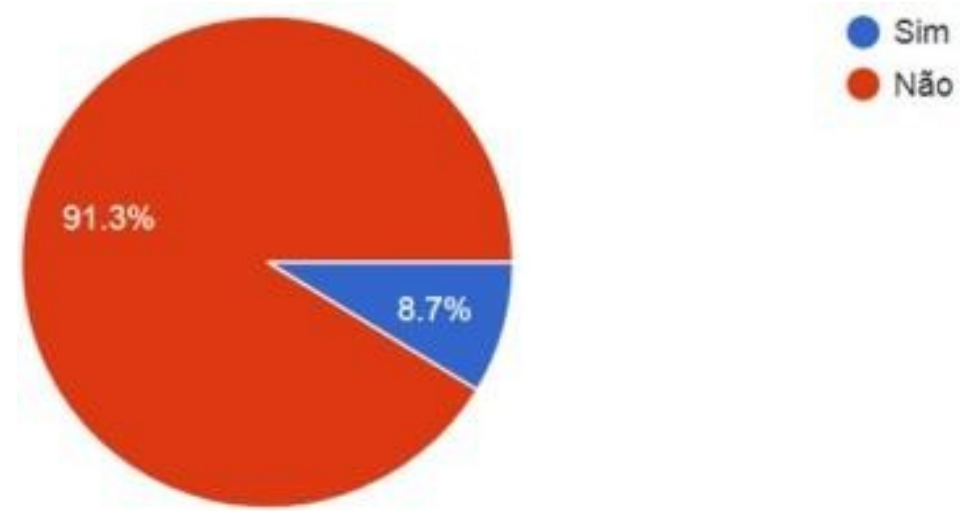

FIGURA 3: Percepção dos egressos do curso de Biotecnologia da UFG sobre a receptividade do mercado de trabalho

Fonte: Elaborado pelos autores com base na aplicação dos questionários

Tal dado é refletido no resultado do questionamento seguinte, acerca da atuação profissional dos participantes, onde apenas um dos egressos entrevistados afirmou estar trabalhando na área de Biotecnologia, ilustrado pela Figura 4.

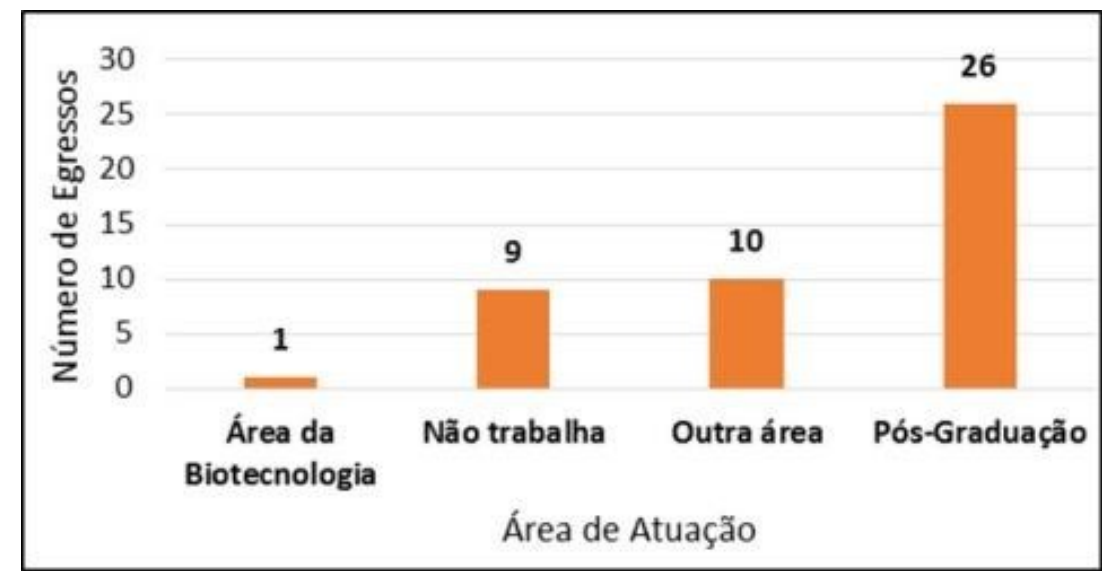

FIGURA 4: Atuação profissional dos egressos do curso de Biotecnologia da UFG.

Fonte: Elaborado pelos autores com base na aplicação dos questionários

O Biotecnologista convive com as dificuldades que uma nova profissão traz, como por exemplo, a falta de reconhecimento e entendimento sobre as competências deste profissional. Sendo assim, a inserção dos egressos no mercado de trabalho ainda é um grande desafio, pois a maioria das empresas e instituições de pesquisa ainda não conhecem seu perfil e habilidades. Devido ao fato de não existir um conselho federal ou regional para dar suporte e garantir o direito dos Biotecnologistas, diversos processos seletivos, como por exemplo, o Concurso Público de Pesquisador Científico Nível I Edital CPRTI 02/2017 (APTA, 2017), são abertos com vagas para realização de atividades que fazem parte da formação do Biotecnologista, mas não contemplam este profissional em seus editais.

Quanto aos locais de atuação dos egressos em Biotecnologia, a grande maioria dos entrevistados afirmou estar em universidades como estudantes de pósgraduação, enquanto apenas $10 \%$ foram para instituições públicas de pesquisa e apenas uma pessoa entre todos os egressos entrevistados investiu em Empreendedorismo e abriu sua própria empresa, como mostrado na Figura 5. 


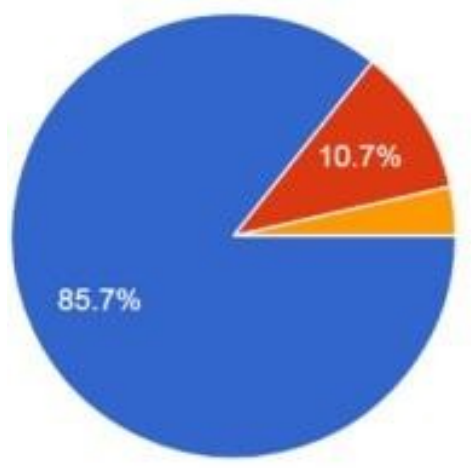

Acadêmica

Empresarial/Örgão público de pesquisa

Abri minha própria empresa

FIGURA 5: Órgãos e instituições que acolheram os egressos do curso de Biotecnologia da UFG.

Fonte: Elaborado pelos autores com base na aplicação dos questionários

De acordo com os entrevistados, atualmente o Biotecnologista segue para a pós-graduação e para a carreira acadêmica, pois o mercado de trabalho e as empresas não são receptivos a esse profissional. Muitos podem escolher por sua vontade seguir para a pós-graduação, porém, o ponto é que profissionais formados e qualificados, como os Biotecnologistas, deveriam ter mais oportunidades de trabalho em sua área específica, sem a necessidade de possuir uma pós-graduação, assim como ocorre em outras profissões: em uma pesquisa realizada em 2017 pelo Sindicato das Entidades Mantenedoras de Estabelecimentos de Ensino superior no Estado de São Paulo (SEMESP) egressos de diversas áreas (Ciências Sociais, Negócios, Direito, Saúde e Bem-estar, Matemática e Computação) foram entrevistados e foi constatado que de cada três alunos, dois estão trabalhando - a maioria em sua área de formação e sem ter realizado pós-graduação, fato que não acontece com os profissionais em Biotecnologia.

As áreas escolhidas para a realização da pós-graduação são bem diversificadas, com destaque para Microbiologia (18\%), Imunologia (9\%), Biologia Molecular (9\%), Biotecnologia Vegetal $(9 \%)$ e Parasitologia $(9 \%)$, mas também incluem, em menor proporção, áreas como bioquímica, Biotecnologia ambiental, química analítica, nanotecnologia, células tronco e biomateriais. A razão para a escolha de tais áreas, de acordo com os participantes, é majoritariamente "satisfação pessoal" (61,5\%), seguida de "falta de opções" (23,1\%), conforme mostrado na Figura 6.

\section{Escolha da Área de Pós-Graduação}

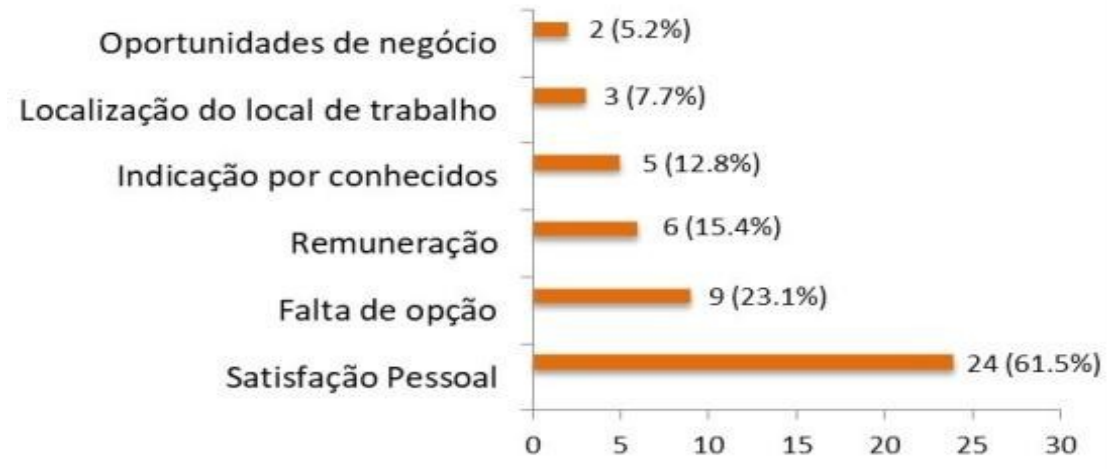

FIGURA 6: Motivos de escolha da área de pós-graduação de acordo com os egressos do curso de Biotecnologia da UFG.

Fonte: Elaborado pelos autores com base na aplicação dos questionários 
Quando se trata da área de especialização escolhida pelos egressos nota-se grande variedade, o que mostra que o curso tem um largo espectro de atuação, possibilitando a participação em várias áreas do conhecimento. A Figura 7 mostra que após o término da graduação, a maioria dos profissionais demorou menos de seis meses para ingressar na pós-graduação $(73.5 \%)$.
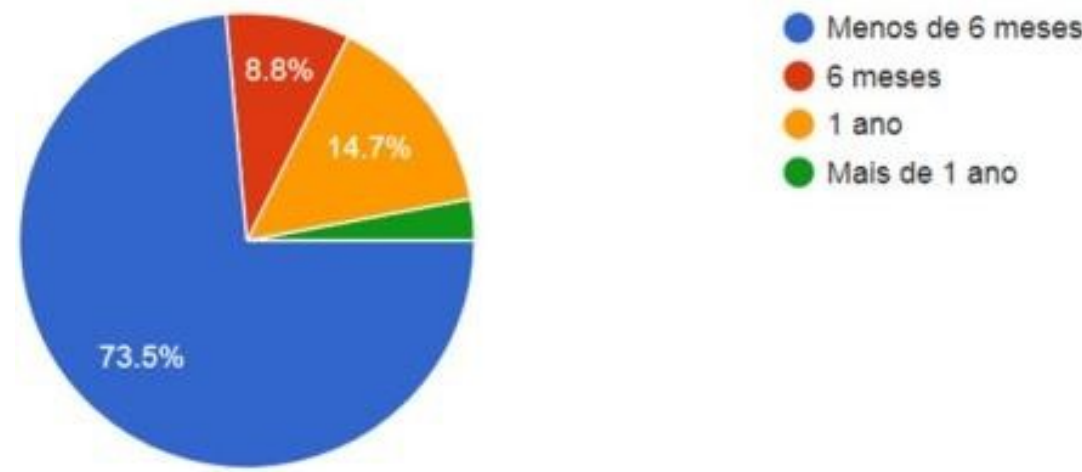

FIGURA 7: Tempo decorrido do fim da graduação até o ingresso na pós-graduação de acordo com os egressos do curso de Biotecnologia da UFG.

Fonte: Elaborado pelos autores com base na aplicação dos questionários

A maioria dos egressos sai da graduação diretamente para o mestrado, muitas vezes ficando no mesmo laboratório onde fizeram iniciação científica ou estágio, adentrando na pós-graduação menos de seis meses após a graduação. Às vezes, por questão de comodidade, ou financeira, os egressos não buscam partir para outras universidades e explorar outras áreas, o que pode explicar os $23,1 \%$ que disseram ter escolhido a sua área de pós-graduação por falta de opção. Segundo Faro (2013), o índice de estresse dos estudantes de pós-graduação no Brasil é mais alto do que em indianos, americanos e nepaleses e pode ser relacionado à baixa renda dos estudantes. A renda de pós-graduandos é em média $\mathrm{R} \$ 1.500,00$, um valor baixo levando em consideração o nível de especialização do profissional. Devido a isso, os estudantes optam por realizar a pós-graduação na cidade em que residem. Também foi constatado que estudantes que se mantiveram na área em que a possuíam experiência mostraram menor índice de estresse durante a pós-graduação.

\section{CONCLUSÃO}

Os dados obtidos mostraram a preocupação sobre a inserção dos Biotecnologistas no mercado de trabalho e as dificuldades enfrentadas por estes profissionais altamente qualificados. Além disso, estes profissionais por ainda enfrentarem muitas dificuldades de emprego partem para dois caminhos: a especialização com uma pós-graduação, pois a Biotecnologia apenas em nível de graduação não se mostra suficiente, ou a mudança drástica de área.

Sendo assim, nota-se a importância de projetos de divulgação e popularização do curso e do profissional em Biotecnologia e também da regularização da profissão. Tais conclusões são úteis tanto para atuais estudantes do curso de Biotecnologia quanto para futuros discentes. Este estudo ainda se faz importante para acompanhar o desenvolvimento de um novo profissional, e a formação de sua trajetória no mercado de trabalho, bem como incentiva políticas 
públicas que valorizem o profissional em Biotecnologia e o desenvolvimento da pesquisa, inovação e empreendedorismo no Brasil.

\section{REFERÊNCIAS}

ABDI. Agência Brasileira de Desenvolvimento Industrial. Panorama da Biotecnologia no Mundo e no Brasil. 2009. Disponível em: <http://www.abdi.com.br/Estudo/Panorama\%20Setorial\%20Biotecnologia.pdf>. Acesso em: 17 fev. 2018

APTA. Agência Paulista de Tecnologia dos Agronegócios. Edital CPRTI 02/2017 "Concurso Público de Pesquisador Científico Nível I". 2017. Disponível em: <http://www.apta.sp.gov.br/concurso/EditalPqC_APTA2017.pdf>. Acesso em: 02 mar. 2018

BRASIL. Projeto de Lei $n^{\circ} 3747,25$ de novembro de 2015, que dispõe sobre regulamentação da profissão de Biotecnologista e cria os Conselhos Federais e Regionais de Biotecnologia. Portal da Câmara dos Deputados. Disponível em: < http://www.camara.gov.br/proposicoesWeb/fichadetramitacao;jsessionid=CFDBFDE6 21BBADBE73D973E59910D1BE.proposicoesWeb1? idProposicao=2056525\&ord=0 >. Acesso em: 25 abr. 2018

BRUNACCI, A. Biotecnologia é setor prioritário, diz Furlan. 2005. Disponível em: $<$ http://investimentos.mdic.gov.br/portalmdic//sitio/interna/noticia.php? area=2\&noticia=6796>. Acesso em: 04 de jan. 2018

DE OLIVEIRA, F. A., DOS SANTOS, A. H., FERNANDES, E.K., CELLES, M. R., AMARAL A. C., et.al. Projeto Pedagógico Do Curso De Graduação Em Biotecnologia. Universidade Federal De Goiás Instituto De Patologia Tropical E Saúde Pública Curso De Graduação Em Biotecnologia. 2017

ERNST \& YOUNG. Biotechnology Industry Report. 2014. Disponível em: <http://www.ey.com/Publication/vwLUAssets/EY-beyond-borders-unlocking-value/ \$FILE/EY-beyond-borders-unlocking-value.pdf>. Acesso em: 10 jan. 2018

ESPARTEL, L. B. O uso da opinião dos egressos como ferramenta de avaliação de cursos: o caso de uma Instituição de Ensino Superior Catarinense. Revista Alcance, Itajaí, v. 16, n. 1, p. 102-114, 2009. ISSN 1983-716X. Disponível em: < http://dx.doi.org/10.14210/alcance.v16n1.p102-114>.

DOI: 10.14210/alcance.v16n1.p102-114

FARO, A. Estresse e estressores na pós-graduação: estudo com mestrandos e doutorandos no Brasil. Psicologia: Teor. e Pesquisas., Brasília, v. 29, n. 1, p. 5160, Mar. 2013. Disponível em http://dx.doi.org/10.1590/S010237722013000100007. DOI: 10.1590/S0102-37722013000100007

LETA, J. As mulheres na ciência brasileira: crescimento, contrastes e um perfil de sucesso. Estudos Avançados, São Paulo, v. 17, n. 49, p.271-284, dez.2003. Dísponivel em: <http://dx.doi.org/10.1590/s0103-40142003000300016>. DOI: $10.1590 / \mathrm{s} 0103-40142003000300016$ 
LiNABIOTEC. Liga Nacional dos Acadêmicos em Biotecnologia. LiNa UFPA. 2016. Disponível em: <https://cabiotecufpa.wixsite.com/biotec/lina-biotec>. Acesso em: 02 mar. 2018

OECD. Organisation for Economic Co-operation and Development. Biotechnology Update. $\quad$ No. 27.2014 .2 Disponivel <http://www.oecd.org/science/biotrack/Biotech-Update-No27-4-July-2014.pdf>. Acesso em: 17 fev. 2018

PINSONNEAULT, A. e KRAEMER, K. L. Survey research methodology in management information system: an assessment. Journal of Management Information Systems, v.10, n.2, Autumn, p.75-105. 1993. Disponível em: $<$ http://dx.doi.org/10.1080/07421222.1993.11518001>. 10.1080/07421222.1993.11518001.

SEMESP. Sindicato das Entidades Mantenedoras de Estabelecimentos de Ensino superior no Estado de São Paulo. DADOS DE EMPREGABILIDADE DE FORMADOS NO ENSINO SUPERIOR. 2017. Disponivel em:<http://www.semesp.org.br/noticias/semesp-apresenta-dados-deempregabilidade-de-formados-no-ensino-superior/>.Acesso em: 26 abr. 2018. 